\title{
475817 - ULTRASOUND-GUIDED SUPRACLAVICULAR BLOCK: INITIAL EXPERIENCE
}

\author{
Kathleen Doyle, MD, Ban Tsui, MD, MSc, FRCPC \\ Anesthesiology and Pain Medicine, University of Alberta, Edmonton, AB, Canada
}

Introduction: Despite their rapid onset and predictable anesthesia, concern about pneumothorax during supraclavicular blockade introduces uncertainty over their suitability for day-case surgery.(1) These blocks have also been associated with procedural difficulty since landmark-based techniques can be inadequate (e.g. with high body mass index [BMI] or anatomical variation).(2) The primary objective of this study was to assess the success rate, block completion time, and postoperative analgesia during our initial experience with combined ultrasound and nerve stimulation-guided supraclavicular blocks (USNSSB) for surgical anesthesia in upper extremity day-case surgery. We also evaluated the impact of BMI and the block-related experience of the anesthesia provider on the outcomes of success and block performance times.

Methods: With REB approval, charts from consecutive patients who received USNSSB during an eight-month period (approximately our first 100 patients) were reviewed. Our US-guidance technique is modified somewhat from that previously described (3), by using a small footprint curved array probe $(\mathrm{C} 11,11 \mathrm{~mm}, 8-5 \mathrm{MHz}$; TITAN or MicroMaxx, SonoSite Inc., Bothell, WA), the footprint enabling good surface contact in the compact supraclavicular fossa and the curved array maximizing needle visibility by improving the US beam incidence angle onto the tangentially angled (generally $45^{\circ}$ ) needle. Additionally, a somewhat anteroposterior needle direction may reduce the inherent risk of unintentional pleural puncture.(4) Blocks were performed by a supervised resident/fellow. Light sedation (midazolam 2 - 5mg, with or without a remifentanil/propofol infusion enabling verbal contact) was given at the outset. Local anesthetic consisted of a $20-30 \mathrm{ml}$ of a mixed lidocaine $(1.5 \%)$ and bupivacaine $(0.125 \%)$ solution, which was injected after US-confirmation of needle-nerve proximity and anesthetic spread. Charts were evaluated for block success (no conversion to general anesthesia), block completion time (procedural time and block onset), recovery time, post-block analgesia requirements and acute complications. The impact of BMI $(>25$ [overweight] vs $\leq 25$ [normal weight]) and the experience of the block proceduralist were evaluated. Data are represented as proportions or means \pm SD and compared by either Chi squared or t-test analysis, respectively ( $\mathrm{P}<0.05$ significant).

Results: The overall block success rate was $94.2 \%$ and block completion times were 20.2 $\pm 9.2 \mathrm{~min}$. There was no significant difference between success rates or block completion times for overweight versus normal weight patients. The operator's procedure-related experience did not significantly affect success rates or block completion times. Recovery room time was $34.7 \pm 5.6 \mathrm{~min}$ and the rate of successful postoperative analgesia was $85.6 \%$. There was no incidence of clinically overt pneumothorax or intra-arterial injection.

Discussion: We conclude that our initial experience with using ultrasound-guided supraclavicular blockade for day surgery has enabled high success for patients of all body 
habitus and without the need for extensive training.

References: 1) Anesth Analg. 1993; 76: 530-4, 2) Anesth Analg. 2006; 102: 1252-4, 3)

Anesth Analg 2003; 97: 1514-7, 4) Anesthesiology 2004; 100: 1325 